\title{
UNRAMIFIED CORRESPONDENCES
}

by

\author{
Fedor Bogomolov and Yuri Tschinkel
}

\begin{abstract}
We study correspondences between algebraic curves defined over algebraic closures of $\mathbb{Q}$ and $\mathbf{F}_{p}$.
\end{abstract}

\section{Contents}

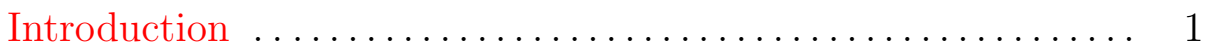

2. Main construction .............................. 3

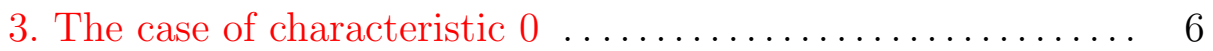

4. Geometric constructions ................... 8

References ................................ 12

\section{Introduction}

A class $\mathcal{C}(\overline{\mathbb{Q}})$ of complete algebraic curves over $\overline{\mathbb{Q}}$ will be called dominating if for every algebraic curve $C^{\prime}$ over $\overline{\mathbb{Q}}$ there exist a curve $\tilde{C} \in \mathcal{C}(\overline{\mathbb{Q}})$ and a surjective map $\tilde{C} \rightarrow C^{\prime}$. A curve $C$ will be called universal if the class $\mathcal{U}_{C}(\overline{\mathbb{Q}})$ of its unramified covers is dominating.

THEOREM 1.1 (Belyi). - Every algebraic curve $C$ defined over a number field admits a surjective map onto $\mathbb{P}^{1}$ which is unramified outside $(0,1, \infty)$.

In 1978 Manin pointed out that Belyi's theorem implies the following 
Proposition 1.2. - The class $\mathcal{M U}(\overline{\mathbb{Q}})$ consisting of modular curves and their unramified covers is dominating.

There are many other classes of curves with the same property, for example:

1. hyperelliptic curves and their unramified coverings;

2. the class $\mathcal{C U}(\overline{\mathbb{Q}}):=\cup_{n \in \mathbb{N}} \mathcal{C}_{n}(\overline{\mathbb{Q}})$, with $\mathcal{C}_{n}(\overline{\mathbb{Q}})$ consisting of curves with function field $\overline{\mathbb{Q}}(z, \sqrt[n]{z(1-z)})$ and their unramified coverings.

3. the class $\mathcal{C N}(\overline{\mathbb{Q}}):=\cup_{n \in \mathbb{N}} \mathcal{C N}_{n}(\overline{\mathbb{Q}})$ where $\mathcal{C N}_{n}(\overline{\mathbb{Q}})$ consists of all unramified covers of any curve $C_{n}$ with the property that $C_{n} \rightarrow \mathbb{P}^{1}$ is ramified in $(0,1, \infty)$ only and all local ramification indices of $C_{n}$ over 0 are divisible by 3 , over 1 divisible by 2 and over $\infty$ divisible by $n$. In particular, we could take $C_{n}$ to be the modular curve $X(n)$.

Proof. - (Sketch) Let us consider the class of hyperelliptic curves and their unramified covers. Let $C^{\prime}$ be an arbitrary curve and $\sigma: C^{\prime} \rightarrow \mathbb{P}^{1}$ a generic map, branched over the points $q_{1}, \ldots, q_{n}$ (generic means that there is only one ramification point over each branch point and all local ramification indices are equal to 2). Denote by $C$ a hyperelliptic curve whose ramification contains $q_{1}, \ldots, q_{n}$. Then $\tilde{C}:=C \times_{\mathbb{P}^{1}} C^{\prime}$ is an unramified cover of $C$ which surjects onto $C^{\prime}$. For the classes $\mathcal{C U}(\overline{\mathbb{Q}})$ and $\mathcal{C N}(\overline{\mathbb{Q}})$ we use Belyi's theorem.

Question 1.3. - Does there exist a universal algebraic curve $C$ (over $\overline{\mathbb{Q}})$ ?

Question 1.4. - Does there exist a number $n \in \mathbb{N}$ such that every curve defined over $\overline{\mathbb{Q}}$ admits a surjective map onto $\mathbb{P}^{1}$ with ramification over $(0,1, \infty)$ such that all local ramification indices are $\leq n$ ?

Question 1.5. - Is every curve $C$ (over $\overline{\mathbb{Q}}$ ) of genus $g(C) \geq 2$ universal?

REMARK 1.6. - It is clear that an affirmative answer to Question 1.4 implies a (constructive) affirmative answer to Question 1.3.

In this note we answer these questions in a simple model situation: instead of $\overline{\mathbb{Q}}$ we consider the (separable) closure $\bar{F}_{p}$ of the finite field $\mathbf{F}_{p}$. 
THEOREM 1.7. - Let $p \geq 5$ be a prime and $C$ a hyperelliptic curve over $\overline{\mathbf{F}}_{p}$ of genus $g(C) \geq 2$. Then $C$ is universal: for any projective curve $C^{\prime}$ there exist a finite étale cover $\tilde{C} \rightarrow C$ and a surjective regular $\operatorname{map} \tau: \tilde{C} \rightarrow C^{\prime}$.

In Section 4 we prove the following geometric fact (over arbitrary algebraically closed fields of characteristic $\neq 2,3$ ):

Proposition 1.8. - Every hyperelliptic curve $C$ of genus $\geq 2$ has a finite étale cover $\tilde{C}$ which surjects onto the genus 2 curve $C_{0}$ given by $\sqrt[6]{z(1-z)}$. In particular, if $C_{0}$ is universal then every hyperelliptic curve of genus $\geq 2$ is universal.

REMARK 1.9. - Applying the Chevalley-Weil theorem we conclude that the Mordell conjecture (Faltings' theorem) for $C_{0}$ implies the Mordell conjecture for every hyperelliptic curve of genus $\geq 2$.

The fact that there is some interaction between the arithmetic of different curves has been noted previously. Moret-Bailly and Szpiro showed (see [6], [5]) that the proof of an effective Mordell conjecture for one (hyperbolic) curve (for example, $C_{0}$ ) implies the ABC-conjecture, which in turn implies an effective Mordell conjecture for all (hyperbolic) curves (Elkies [4]). Here effective means an explicit bound on the height of a $K$-rational point on the curve for all number fields $K$. Here again, Belyi's theorem is used in an essential way.

Acknowledgments. We have benefited from conversations with B. Hassett and A. Chambert-Loir. The first author was partially supported by the NSF. The second author was partially supported by the NSF and the Clay foundation.

\section{Main construction}

Notations 2.1. - Let $\tau: C \rightarrow C^{\prime}$ be a surjective map of algebraic curves. We denote by $\operatorname{Ram}(\tau) \subset C$ the ramification locus of $\tau$ and by $\operatorname{Bran}(\tau)=\tau(\operatorname{Ram}(C)) \subset C^{\prime}$ the branch locus of $\tau$. For a point $q \in C$ we 
denote by $e_{q}(\tau)$ the local ramification index at $q$. We denote by

$$
e(\tau):=\max _{q \in C} e_{q}(\tau)
$$

the maximum local ramification index of $\tau$. We say that $\tau$ has simple ramification if $e(\tau) \leq 2$ and that $\tau$ is generic if in addition there is only one ramification point over each branch point.

REMARK 2.2. - Every curve admits a generic map onto $\mathbb{P}^{1}$, at least after a separable extension of the ground field.

Let $p \geq 5$ be a prime number. In this section we work over a separable closure $\overline{\mathbf{F}}_{p}$ of the finite field $\mathbf{F}_{p}$. First we show that there exists at least one curve satisfying the conclusion of Theorem 1.7.

Let $\pi_{0}: E_{0} \rightarrow \mathbb{P}^{1}$ be the elliptic curve given by

$$
\sqrt[3]{z(z-1)}
$$

Let $\sigma_{0}: C_{0} \rightarrow \mathbb{P}^{1}$ be the genus 2 curve given by

$$
\sqrt[6]{z(z-1)}
$$

and $\iota_{0}: C_{0} \rightarrow E_{0}$ the corresponding 2-cover. Clearly, $\iota_{0}$ has simple ramifications over the preimages of 0,1 . Let $C$ be an arbitrary curve. Choosing a generic function on $C$ we get a generic covering $\sigma: C \rightarrow \mathbb{P}^{1}$ (such covering is defined over $\overline{\mathbf{F}}_{p}$ ). Assume further that $\operatorname{Bran}(\sigma) \subset \mathbb{P}^{1}$ does not contain $(0,1, \infty)$.

Consider the diagram

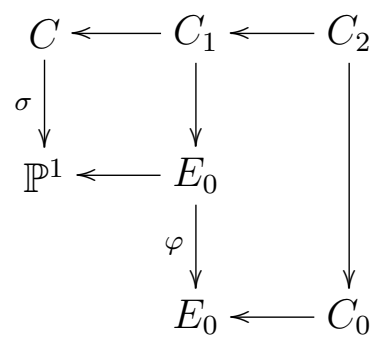

Here $C_{1}=C \times_{\mathbb{P}^{1}} E_{0}$ (it is irreducible since $E_{0} \rightarrow \mathbb{P}^{1}$ is a 2-cover). Then $C_{1} \rightarrow E_{0}$ has simple ramification over a finite number of points in $E_{0}$. Recall that $E_{0}$ has a group scheme structure, and all $\overline{\mathbf{F}}_{p}$-points of $E_{0}$ are torsion points. This implies that there exists an étale map 
$E_{0} \rightarrow E_{0}$ such that all ramification points of $C_{1}$ over $E_{0}$ are mapped to 0 . More precisely, any finite set of $\overline{\mathbf{F}}_{p}$-points of $E_{0}$ is contained in the group subscheme $E_{0}^{e t}[n] \subset E_{0}$ - the maximal étale subgroup of the multiplication by $n$-kernel $E_{0}[n]$ (for some $n \in \mathbb{N}$ ). For every positive integer $n$ there exists a positive multiple of $m$ of $n$ and an étale map $E_{0} \rightarrow E_{0}$ with kernel $E_{0}^{e t}[m]$.

Taking the composition of $C_{1} \rightarrow E_{0}$ with the multiplication by a suitable $m$, we get a (possibly new) surjective regular map $C_{1} \rightarrow E_{0}$ which is ramified only over the zero point in $E_{0}$ and has the property that all the local ramification indices are at most 2. Using this map let us define $C_{2}:=C_{0} \times_{E_{0}} C_{1}$. Consequently, any component of $C_{2}$ surjects onto $C_{1}$ and is an étale covering of $C_{0}$ (ramification cancels ramification). This component satisfies the conclusion of Theorem 1.7.

Lemma 2.3. - Let $C$ be any smooth complete algebraic curve and $E$ any curve of genus 1 . There exists a curve $C_{1}$ which surjects onto $C$ and $E$ such that the ramification of the map $C_{1} \rightarrow E$ lies entirely over a single point of $E$ and its local ramification indices are all equal to 2.

Proof. - Consider a generic map $\sigma: C \rightarrow \mathbb{P}^{1}$ with $e(\sigma) \leq 2$. Choose a double cover $\pi: E \rightarrow \mathbb{P}^{1}$ such that the branch loci $\operatorname{Bran}(\sigma)$ and $\operatorname{Bran}(\pi)$ on $\mathbb{P}^{1}$ are disjoint. Then the product $C_{1}:=C \times_{\mathbb{P}^{1}} E$ is an irreducible curve which is a double cover of $C$. The curve admits a surjective map $\iota_{1}: C_{1} \rightarrow E$ with $e\left(\iota_{1}\right) \leq 2$. Similarly to the previous construction we can find an unramified cover $\varphi: E \rightarrow E$ such that the composition $\varphi \circ \iota_{1}: C_{1} \rightarrow E$ is ramified only over one point in $E$ and the local ramification indices are still equal to 2 .

Corollary 2.4. - Assume that some unramified covering $\tilde{C}$ of $C$ surjects onto an elliptic curve $E$. Assume further that there exists a point $q$ on $E$ such that all local ramification indices of the map $\tilde{C} \rightarrow E$ over $q$ are divisible by 2. Then $C$ is universal.

Proof. - It is sufficient to take the product of $\tilde{C} \times{ }_{E} C_{1}$. Any irreducible component of the resulting curve will be an unramified covering of $\tilde{C}$ (and hence $C$ ) and will admit a surjective map onto $C_{1}$ and $C$. 
Corollary 2.5 (Theorem 1.7). - Every hyperelliptic curve $C$ over $\overline{\mathbf{F}}_{p}$ (with $p \geq 5$ ) of genus $\geq 2$ is universal.

Proof. - Consider the standard projection $\sigma: C \rightarrow \mathbb{P}^{1}$ (of degree 2). Its branch locus $\operatorname{Bran}(\sigma)$ consists of $2 g+2$ points. Let $\pi: E \rightarrow \mathbb{P}^{1}$ be a double cover such that $\operatorname{Bran}(\pi)$ is contained in $\operatorname{Bran}(\sigma)$. Then the product $\tilde{C}=C \times \times_{\mathbb{P} 1} E$ is an unramified double cover of $C$. Moreover, $\tilde{C}$ is a double cover of $E$ with ramification at most over the preimages in $E$ of the points in $\operatorname{Bran}(\sigma) \backslash \operatorname{Bran}(\pi)$. We now apply Corollary 2.4.

In finite characteristic, there are many other (classes of) universal curves. For example, cyclic coverings with ramification in 3 points, hyperbolic modular curves, etc. Thus it seems plausible to formulate the following

Conjecture 2.6. - Any smooth complete curve $C$ of genus $g(C) \geq 2$ defined over $\overline{\mathbf{F}}_{p}$ (for $p \geq 2$ ) is universal.

\section{The case of characteristic 0}

In this section we work over $\overline{\mathbb{Q}}$. We show that the method outlined in Section 2 can employed in characteristic zero to produce natural infinite sets of algebraic points on $\mathbb{P}^{1}$ which occur as ramification points of surjective maps from $\mathbb{P}_{2}^{1}$ to $\mathbb{P}_{1}^{1}$ branched over $(0,1, \infty) \in \mathbb{P}_{1}^{1}$ only and having an a priori bound on the ramification index (here $\mathbb{P}_{1}^{1}$ and $\mathbb{P}_{2}^{1}$ are two different copies of the projective line $\mathbb{P}^{1}$ ).

Notice that, in principle, it is easy to produce some sets of points (of any finite cardinality) with this property: Take an $n \geq 6$ and any triangulation of $\mathbb{P}_{2}^{1}$ with vertices of index $\leq n$. A barycentric subdivision of each such triangulation defines a function from $\mathbb{P}_{2}^{1}$ to $\mathbb{P}_{1}^{1}$ with local ramification indices $\leq 2 n$ (for more details see [3]). Therefore, any curve with bounded ramification over this set of vertices will have bounded ramification over $\mathbb{P}_{1}^{1}$. However, we have no explicit control over the coordinates of the ramification points on $\mathbb{P}_{2}^{1}$.

An (obvious) analogous way to control ramification indices is to consider the following diagram 


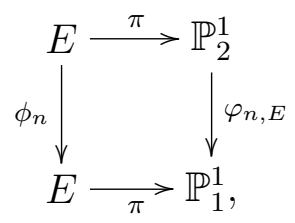

where the map $\phi_{n}$ is the quotient by the subscheme of $n$-torsion points and the maps $E \rightarrow \mathbb{P}^{1}$ are the standard double covers, ramified over $(0,1, \infty, \lambda)$. Clearly, all the ramification points of $\varphi_{n, E}$ (in $\left.\mathbb{P}_{2}^{1}\right)$ are over $0,1, \infty$ and $\lambda$ (in $\mathbb{P}_{1}^{1}$ ) and $e\left(\varphi_{n, E}\right)=2$. Belyi's theorem gives a map $\beta: \mathbb{P}_{1}^{1} \rightarrow \mathbb{P}_{0}^{1}$, which ramifies only over the points $(0,1, \infty) \in \mathbb{P}_{0}^{1}$, maps $\{0,1, \infty, \lambda\} \subset \mathbb{P}_{1}^{1}$ into $\{0,1, \infty\} \subset \mathbb{P}_{0}^{1}$ and has local ramification indices $\leq$ $n$. Moreover, it provides an explicit bound on $\operatorname{deg}(\beta)$ and, consequently, on $e(\beta)$ (in terms of the absolute height of $\lambda$ ). Let $\beta_{\lambda}: \mathbb{P}_{1}^{1} \rightarrow \mathbb{P}_{0}^{1}$ be a map such that

$$
e\left(\beta_{\lambda}\right)=\inf _{\beta}\left\{e_{\beta}\right\}
$$

over the set of all maps as above. Then the map $\beta_{\lambda} \circ \varphi_{n, E}: \mathbb{P}_{2}^{1} \rightarrow \mathbb{P}_{0}^{1}$ ramifies over three points only and has index $e\left(\beta_{\lambda} \circ \varphi_{n, E}\right) \leq 2 n$. Let

$$
R_{E}:=\pi\left(E(\overline{\mathbb{Q}})_{\text {tors }}\right) \subset \mathbb{P}_{2}^{1}(\overline{\mathbb{Q}})
$$

be the image of the torsion points of $E$. Let $\sigma: C \rightarrow \mathbb{P}_{2}^{1}$ be any map ramified only in a subset of $R_{E}$. Let $\pi:=\beta_{\lambda} \circ \varphi_{n, E} \circ \sigma$. Then

$$
e(\pi) \leq 2 e(\sigma) \cdot e\left(\beta_{\lambda}\right) .
$$

A natural application of the construction in Section 2 is as follows:

ExAmple 3.1. - Let $\pi: E \rightarrow \mathbb{P}^{1}$ be a triple cover with $\operatorname{Bran}(\pi)=$ $\{0,1, \infty\}$ ( $E$ is a $\mathrm{CM}$ elliptic curve with $j$-invariant 0$)$. Consider the following diagram

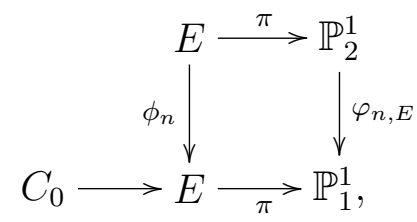


where $C_{0}$ is a curve of genus $g\left(C_{0}\right)=2$ given by $\sqrt[6]{z(z-1)}, \phi_{n}$ is the quotient map by the subscheme of torsion points of order $n$, and $\varphi_{n, E}$ the corresponding map from $\mathbb{P}_{2}^{1}$ to $\mathbb{P}_{1}^{1}$ ramified only over $(0,1, \infty)$. Let $\mathcal{X}_{g}=$ $\{X\}$ be the subset of curves of genus $g$ admitting a map $\sigma_{X}: X \rightarrow \mathbb{P}_{2}^{1}$ such that

$$
\begin{aligned}
& -e\left(\sigma_{X}\right)=2 ; \\
& -\operatorname{Bran}\left(\sigma_{X}\right) \subseteq \pi\left(E(\overline{\mathbb{Q}})_{\text {tors }}\right) .
\end{aligned}
$$

Then, for any $X \in \mathcal{X}_{g}$ the map

$$
\varphi_{n, E} \circ \sigma_{X}: X \rightarrow \mathbb{P}_{1}^{1}
$$

has index $e\left(\varphi_{n, E} \circ \sigma_{X}\right) \leq 6$ and there exists an unramified cover $\tilde{C} \rightarrow C_{0}$ surjecting onto $X$. Moreover, $\mathcal{X}_{g}$ is dense (in real and $p$-adic topologies) in the natural Hurwitz scheme $\mathcal{H}_{g}$ parametrizing curves of genus $g$.

The set of curves dominated by unramified covers of $C_{0}$ is much larger than $\mathcal{X}_{g}$. Indeed, consider any 4 -tuple of points in

$$
\pi\left(E(\overline{\mathbb{Q}})_{\text {tors }}\right) \subseteq \mathbb{P}_{2}^{1}
$$

and an elliptic curve $E^{\prime}$ obtained as a double cover of $\mathbb{P}_{2}^{1}$ ramified in those 4 points. Then $E^{\prime}$ is also dominated by unramified covers of $C_{0}$ and we can iterate the above construction for $E^{\prime}$.

\section{Geometric constructions}

Let $\left(E, q_{0}\right)$ be an elliptic curve, $q_{1}$ a torsion point of order two on $E$ and $\pi: E \rightarrow \mathbb{P}^{1}$ the quotient with respect to the involution induced by $q_{1}$. Let $n$ be an odd positive integer and $\varphi_{n, E}: \mathbb{P}_{2}^{1} \rightarrow \mathbb{P}_{1}^{1}$ the map induced by

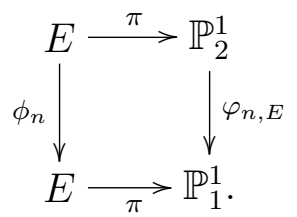

Any quadruple $r=\left\{r_{1}, \ldots, r_{4}\right\}$ of four distinct points in $\varphi_{n, E}^{-1}\left(\pi\left(q_{0}\right)\right)$ defines a genus 1 curve $E_{r}$ (the double cover of $\mathbb{P}^{1}$ ramified in these four points). 
Proposition 4.1. - Let $\iota: C \rightarrow E$ be any finite cover such that all local ramification indices over $q_{0}$ are even. Then there exists an unramified cover $\tau_{r}: C_{r} \rightarrow C$ which dominates $E_{r}$ and has only even local ramification indices over some point in $E_{r}$.

Proof. - Assume that $n \geq 3$ and consider the following diagram

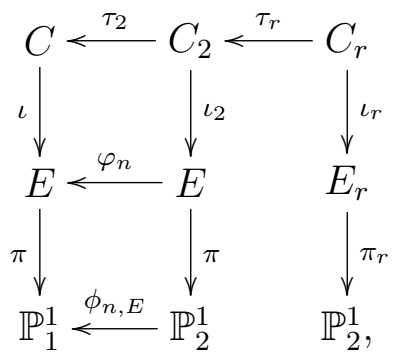

where $E_{r}$ is a double cover of $\mathbb{P}_{2}^{1}$ ramified in any quadruple of points in the preimage $\phi_{n, E}^{-1}\left(\pi\left(q_{0}\right)\right)$ and $C_{r}$ is any irreducible component of $C_{2} \times_{\mathbb{P}_{2}^{1}} E_{r}$. Any point $q_{r} \in E_{r}$ such that $q_{r} \notin \operatorname{Ram}\left(\pi_{r}\right)$ (that is, its image in $\mathbb{P}_{2}^{1}$ is distinct from $\left.r_{1}, \ldots, r_{4}\right)$ has the claimed property.

REMARK 4.2. - Iterating this procedure (and adding isogenies) we obtain many elliptic curves $E^{\prime}$ which are dominated by curves having an unramified cover onto $E$. It would be interesting to know if for any two elliptic curves over $\overline{\mathbb{Q}}$ there exists a cycle connecting them (at least modulo isogenies). We will now show that any elliptic curve can be connected in this way to $E_{0}$.

Let $E_{0} \subset \mathbb{P}^{2}=\{(x: y: z)\}$ be the elliptic curve

$$
x^{3}+y^{3}+z^{3}=0,
$$

and

$$
E_{0}[3]=\mathrm{T}:=\left\{\begin{array}{ccc}
(1: 0:-1), & (1: 0:-\zeta), & \left(1: 0:-\zeta^{2}\right), \\
(0: 1:-1), & (0: 1:-\zeta), & \left(0: 1:-\zeta^{2}\right), \\
(1:-1: 0), & (1:-\zeta: 0), & \left(1:-\zeta^{2}: 0\right)
\end{array}\right\}
$$

its set of 3 -torsion points (where $\zeta$ is a primitive cubic root of 1 ). Denote by $\mathcal{E}_{\lambda}=\left\{E_{\lambda}\right\}$ the family of elliptic curves on $\mathbb{P}^{2}$ passing through $\mathrm{T}$ given by

$$
E_{\lambda}: x^{3}+y^{3}+z^{3}+\lambda x y z=0 .
$$


It is easy to see that for each $\lambda$ the set $E_{\lambda}[3]$ of 3-torsion points of $E_{\lambda}$ is precisely $\mathrm{T}$. Let

$$
\begin{array}{lllc}
\pi: & \mathbb{P}^{2} & \rightarrow & \mathbb{P}^{1} \\
(x: y: z) & \mapsto & (x+z: y)
\end{array}
$$

be the projection respecting the involution $x \rightarrow z$ on $\mathbb{P}^{2}$. Denote by $E_{\lambda}^{0}=E_{\lambda} \backslash(1: 0:-1)$ and by $\pi_{\lambda}$ the restriction of $\pi$ to $E_{\lambda}^{0}$. Clearly, $\pi_{\lambda}$ exhibits each $E_{\lambda}^{0}$ as a double cover of $\mathbb{P}^{1}$. and $\pi_{\lambda}$ has only simple double points for all $\lambda$. Moreover,

$$
\pi(\mathrm{T} \backslash(1: 0:-1))=\left\{(1:-\zeta),\left(1:-\zeta^{2}\right),(1:-1),(1: 0)\right\}
$$

and for all $\lambda$ there exists a (non-empty) set $S_{\lambda} \subset \operatorname{Bran}\left(\pi_{\lambda}\right) \subset \mathbb{P}^{1}$ such that $\pi_{\lambda}^{-1}\left(S_{\lambda}\right) \subset \mathrm{T}$. Let $\pi_{0}^{\prime}: E_{0}^{\prime} \rightarrow \mathbb{P}^{1}$ be a double cover ramified in the 4 points in $\pi(\mathrm{T} \backslash(1: 0:-1))$.

Lemma 4.3. - Let $\iota: C \rightarrow E_{\lambda}$ be a double cover such that over at least one point in $\operatorname{Bran}(\iota)$ the local ramification indices are even. Then there exists an unramified cover $\tilde{C} \rightarrow C$ and a surjective morphism $\tilde{\iota}: \tilde{C} \rightarrow E_{0}^{\prime}$ such that over at least one point in $\operatorname{Bran}(\tilde{\iota}) \subset E_{0}^{\prime}$ all local ramification indices of $\tilde{\iota}$ are even.

Proof. - Consider the diagram

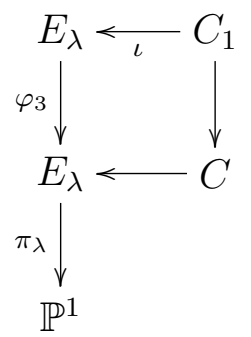

Then $C_{1} \rightarrow \mathbb{P}^{1}$ has even local ramification indices over all points in $\pi(\mathrm{T})$. It follows that

$$
\tilde{C}:=C_{1} \times_{\mathbb{P}^{1}} E_{0}^{\prime} \rightarrow E_{0}^{\prime}
$$

has even local ramification indices over the preimages of the fifth point in $\pi(\mathrm{T})$, as claimed. 
Notations 4.4. - Let $\mathcal{C}$ be the class of curves such that there exists an elliptic curve $E$, a surjective map $\iota: C \rightarrow E$ and a point $q \in \operatorname{Bran}(\iota)$ such that all local ramification indices at points in $\iota^{-1}(q)$ are even.

EXAmple 4.5. - Any hyperelliptic curve of genus $\geq 2$ belongs to $\mathcal{C}$. More generally, $\mathcal{C}$ contains any curve $C$ admitting a map $C \rightarrow \mathbb{P}^{1}$ with even local ramification indices over at least 5 points in $\mathbb{P}^{1}$.

Proposition 4.6. - For any $C \in \mathcal{C}$ there exists an unramified cover $\tilde{C} \rightarrow C$ surjecting onto $C_{0}$ (with $C_{0} \rightarrow \mathbb{P}^{1}$ given by $\sqrt[6]{z(1-z)}$ ).

Proof. - Consider $C_{1}=C \in \mathcal{C}$ with $\iota_{1}: C_{1} \rightarrow E=E_{\lambda}$ as in 4.4. Define $C_{2}$ as an irreducible component of $C_{1} \times_{E} E$ :

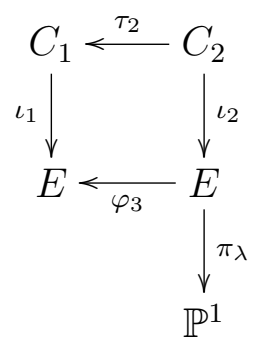

Define $C_{3}:=C_{2} \times_{\mathbb{P}^{1}} E_{0}$ by the diagram

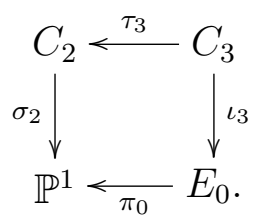

Observe that for $q \in \operatorname{Bran}\left(\pi_{0}\right)$ the local ramification indices in the preimage $\left(\pi_{\lambda} \circ \iota_{2}\right)^{-1}(q)$ are all even. It follows that the map $\tau_{3}: C_{3} \rightarrow C_{2}$ is unramified and that $\iota_{3}: C_{3} \rightarrow E_{0}$ has even local ramification indices over (the preimage of) $q_{5} \in\left\{\pi(\mathrm{T}) \backslash \operatorname{Bran}\left(\pi_{0}\right)\right\}$ (the 5 th point). Define $C_{4}$ as an irreducible component of $C_{3} \times_{E_{0}} E_{0}$ in the diagram 


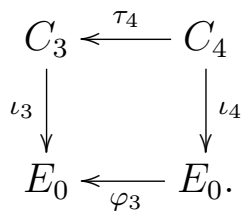

The map $\iota_{4}$ is ramified over the preimages $\left(\pi_{0} \circ \varphi_{3}\right)^{-1}\left(q_{5}\right)$, with even local ramification indices. Finally, $C_{5}=C_{4} \times_{E_{0}} C_{0}$ from the diagram

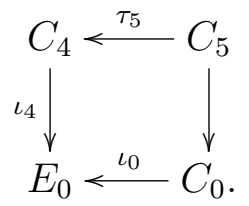

has a dominant map onto $C_{0}$ and is unramified over $C_{4}$ (and consequently, $\left.C_{1}\right)$.

\section{References}

[1] G. V. Belyi, Galois extensions of a maximal cyclotomic field, Izv. Akad. Nauk SSSR Ser. Mat. 43, (1979), no. 2, 267-276, 479.

[2] G. V. Belyi, Another proof of the Three Points theorem, Preprint MPI 1997-46, (1997).

[3] F. Bogomolov, D. Husemoller, Geometric properties of curves defined over number fields, Preprint MPI 2000-1, (2000).

[4] N. Elkies, ABC implies Mordell, Intern. Math. Res. Notices 7, (1991), 99-109.

[5] L. Moret-Bailly, Hauteurs et classes de Chern sur les surfaces arithmétiques, Astérisque 183, (1990), 37-58.

[6] L. Szpiro, Discriminant et conducteur des courbes elliptiques, Astérisque 183, (1990), 7-18. 